\title{
EDITORIAL \\ Daily neurosurgical experiences with ethics and the elderly
}

\author{
Mark G. Hamilton, MDCM \\ Division of Neurosurgery, and Adult Hydrocephalus Program, Department of Clinical Neurosciences, University of Calgary, \\ Alberta, Canada
}

$\mathrm{T}$ HE paper by Hachem and Bernstein ${ }^{1}$ addressing ethical issues in geriatric cranial neurosurgery is an articulate and balanced outline of the important ethical challenges frequently faced in neurosurgery, but unduly so during care of the elderly or geriatric population. Four main principles are outlined: autonomy, beneficence, nonmaleficence, and justice. It should be required reading for all neurosurgeons.

The following summary cannot do justice to Hachem and Bernstein's eloquent paper. Rather, it is meant to highlight the key principles that should be considered and to provide some additional clinical context. Age-related changes in the physiology of the brain, CNS and non-CNS comorbidities, and associated frailty generate increased vulnerability, skew the severity of the underlying disease, and adversely affect expectations for response to treatment and resultant outcomes. However, to respect these geriatric principles does not mean that treatment should be withheld, but rather that we must acknowledge these issues and strive for balance.

The four well-established ethical principles discussed by Hachem and Bernstein ${ }^{1}$ include:

1) Personal autonomy, which is the capacity to decide for oneself and pursue a course of action in one's life. However, in many situations, an elderly patient may be unable to effectively convey their goals of care, and decisions regarding treatment may be undertaken without full awareness of expressed or unexpressed wishes. In addition, there may be circumstances in which conflict exists with family members regarding who can represent the "best interests" of the patient. A sensible goal should be to remember in whom your care as a neurosurgeon is vested.

2) Beneficence is the moral obligation to do good and the moral obligation to act for the other's benefit.

3) Nonmaleficence is strongly linked to beneficence and is best summarized by the phrase "first do no harm." This should not imply, for instance, that withholding a treatment such as surgery is a necessary pathway, but rather that judgment should be used regarding real risks and true benefit.
4) Lastly, justice, which implies a moral obligation to act with fairness such that each person gets their due. This also has implications with regards to expectations and goals of care, patient engagement in care, and healthcare resources and health economics.

Neurosurgeons predictably engage with these ethical principles during daily clinical care. Recently, a neurosurgery resident questioned what it would take to be more comfortable and confident regarding some of these difficult clinical decisions. The solution lies with trying to leverage these ethical principles to guide oneself through the process. For many situations, it will probably never be easy, but if approached with a respect for the described ethical principles and if undertaken with sincerity and honesty, it will be less difficult and ultimately more gratifying.

For context, a few acute and nonacute examples from a week of on-call experience are briefly described below. While simplified in detail, these examples illustrate themes common to the current geriatric neurosurgical experience. While for some of these stories the pathway may seem obvious, no resultant actions or outcomes are provided. It is important to remember that the key elements relevant to geriatric neurosurgical care outlined in the editorial by Schmidt et al. ${ }^{2}$ are pertinent and that chronological age is an incomplete biomarker when used alone to predict outcome.

\section{Acute Cases}

A 68-year-old patient with a mechanical mitral valve treated with warfarin had a ground-level fall resulting in a small acute subdural hemorrhage. His warfarin was stopped. The patient experienced a large right middle cerebral artery stroke on day 3 with hemiplegia but remained alert. Interventional radiology was unsuccessful. The initial goals of care include all options. When is it appropriate to restart the anticoagulant? Is a decompressive craniotomy a reasonable option if this patient experiences the clinical effects of cerebral swelling (balancing 
the risks and benefits of anticoagulation if that option is undertaken)? Should the goals of care be reevaluated?

A 70-year-old patient presents with a large (7-cm maximal diameter) left frontotemporal intracerebral hemorrhage (ICH). The patient was taking a direct oral anticoagulant due to a diagnosis of atrial fibrillation. The initial goals of care include all options. The patient has a dense right hemiplegia and is aphasic, and the pupils are reactive. Should surgery to remove the ICH be offered? Should the goals of care be reevaluated?

A 65 -year-old patient presents with a large $(8-\mathrm{cm}$ maximal diameter) left frontotemporal ICH. The goals of care are unknown. There is no evidence of anticoagulant use. The patient's Glasgow Coma Scale (GCS) score is 5 (with right hemiplegia) and the left pupil is fixed and dilated. Should surgery to remove the ICH be offered? Should the goals of care be reevaluated?

An 85-year-old patient presents with a large $(3.5-\mathrm{cm}$ maximal diameter) central cerebellar hemorrhage with mild obstructive hydrocephalus. The patient was taking coumadin due to a diagnosis of atrial fibrillation (international normalized ratio 4.6) and has a history of moderate dementia. The initial goals of care include all options. The patient's GCS score is 13. Should surgery to remove the cerebellar hematoma be offered? Should an external ventricular drain be considered? Should the goals of care be reevaluated?

\section{Nonacute Cases}

An 80-year-old patient with cognitive issues was falling down and underwent MRI that demonstrated features consistent with a glioblastoma of the posterior (splenium) corpus callosum. The initial goals of care include all options. Should a biopsy be offered? Should dexamethasone be used? Should the goals of care be changed? How would this be different with or without the involvement of a family member?
A 90-year-old patient with a large (6-cm maximal diameter) left lateral frontal-parietal extraaxial tumor consistent with a diagnosis of meningioma presented with speech and walking difficulties. He had been treated elsewhere with a corticosteroid for a later discounted diagnosis, but which was associated with a significant improvement in his symptoms. He has minimal medical comorbidities. Should surgery be offered? How should the discussion regarding the goals of surgery, as well as the risks and benefits of surgery (the consent process), be framed? How would this be different with or without the involvement of a family member?

As you read the papers that follow and examine reports addressing the clinical and neurological conditions that are encountered in the elderly, endeavor to allow the four ethical principles to provide the foundation for your journey.

https://thejns.org/doi/abs/10.3171/2020.7.FOCUS20667

\section{References}

1. Hachem LD, Bernstein M. Ethical issues in geriatric cranial neurosurgery. Neurosurg Focus. 2020;49(4):E3.

2. Schmidt E, Balardy L, Geeraerts T, et al. Editorial. Geriatric neurosurgery: the unfolding of a new subspecialty. Neurosurg Focus. 2020;49(4):E2.

\section{Disclosures}

The author reports no conflict of interest.

\section{Correspondence}

Mark G. Hamilton: mghamilton.hydro@gmail.com.

INCLUDE WHEN CITING

DOI: 10.3171/2020.7.FOCUS20667. 